\title{
Optimising the utility of pleural fluid adenosine deaminase for the diagnosis of adult tuberculous pleural effusion in Hong Kong
}

\author{
KC Chang *, MC Chan, WM Leung, FY Kong, Chloe M Mak, Sammy PL Chen, WC Yu
}

\section{A B S T R A C T}

Introduction: Pleural fluid adenosine deaminase level can be applied to rapidly detect tuberculous pleural effusion. We aimed to establish a local diagnostic cut-off value for pleural fluid adenosine deaminase to identify patients with tuberculous pleural effusion, and optimise its utility.

Methods: We retrospectively reviewed the medical records of consecutive adults with pleural fluid adenosine deaminase level measured by the Diazyme commercial kit (Diazyme Laboratories, San Diego [CA], United States) during 1 January to 31 December 2011 in a cluster of public hospitals in Hong Kong. We considered its level alongside early (within 2 weeks) findings in pleural fluid and pleural biopsy, with and without applying Light's criteria in multiple scenarios. For each scenario, we used the receiver operating characteristic curve to identify a diagnostic cut-off value for pleural fluid adenosine deaminase, and estimated its positive and negative predictive values.

Results: A total of 860 medical records were reviewed. Pleural effusion was caused by congestive heart failure, chronic renal failure, or hypoalbuminaemia caused by liver or kidney diseases in 246 (28.6\%) patients, malignancy in 198 (23.0\%), non-tuberculous infection in $168(19.5 \%)$, tuberculous pleural effusion in 157 (18.3\%), and miscellaneous causes in 91 (10.6\%). All those with tuberculous pleural effusion had a pleural fluid adenosine deaminase level of $\leq 100 \mathrm{U} / \mathrm{L}$. When analysis was restricted to 689 patients with pleural fluid adenosine deaminase level of $\leq 100 \mathrm{U} / \mathrm{L}$ and early negative findings for malignancy and non-tuberculous infection in pleural fluid, the positive predictive value was significantly increased and the negative predictive neither additionally restricting analysis to exudates by Light's criteria nor adding closed pleural biopsy would further enhance predictive values. As such, the diagnostic cut-off value for pleural fluid adenosine deaminase is $26.5 \mathrm{U} / \mathrm{L}$, with a sensitivity of $87.3 \%$, specificity of $93.2 \%$, positive predictive value of $79.2 \%$, negative predictive value of $96.1 \%$, and accuracy of 91.9\%. Sex, age, and co-morbidity did not significantly affect prediction of tuberculous pleural effusion using the cut-off value.

Conclusion: We have established a diagnostic cut-off level for pleural fluid adenosine deaminase in the diagnosis of tuberculous pleural effusion by restricting analysis to a level of $\leq 100 \mathrm{U} / \mathrm{L}$, and considering early pleural fluid findings for malignancy and nontuberculous infection, but not Light's criteria.
Hong Kong Med J 2018;24:38-47
DOI: $10.12809 / \mathrm{hkmj} 176238$
${ }^{1}$ KC Chang ${ }^{*}$, MSc, FHKAM (Medicine)
${ }^{2}$ MC Chan, MB, BS, MRCP (UK)
${ }^{1}$ WM Leung, MB, ChB, FHKAM (Medicine)
${ }^{3}$ FY Kong, MB, BS, FHKAM (Medicine)
${ }^{4}$ CM Mak, MD, FHKAM (Pathology)
${ }^{4}$ SPL Chen, MRes(Med), FHKAM (Pathology)
${ }^{2}$ WC Yu, FRCP, FHKAM (Medicine)

\section{Tuberculosis and Chest Service, Department of Health, Hong Kong Department of Medicine and Geriatrics, Princess Margaret Hospital, Laichikok, Hong Kong \\ Department of Medicine and Geriatrics, Yan Chai Hospital, Tsuen Wan, Hong Kong \\ Chemical Pathology Laboratory, Department of Pathology, Princess Margaret Hospital, Laichikok, Hong Kong}

* Corresponding author: kc_chang@dh.gov.hk

New knowledge added by this study

- There are limitations to the use of pleural fluid adenosine deaminase (pfADA) level as a surrogate marker for tuberculous pleural effusion (TBPE); thus, it must be interpreted alongside other findings that help exclude nontuberculous diseases, thereby increasing the pre-test probability of TBPE and the positive predictive value (PPV). We demonstrated that TBPE was unlikely when pfADA level was $>100 \mathrm{U} / \mathrm{L}$.

- Restricting analysis to patients with pfADA level of $\leq 100 \mathrm{U} / \mathrm{L}$ and early (within 2 weeks) negative findings for malignancy and non-tuberculous infection in pleural fluid significantly increased PPV and non-significantly reduced the negative predictive value (NPV). Using this approach, neither additionally restricting analysis to exudates by Light's criteria nor adding closed pleural biopsy would further enhance predictive values of pfADA for TBPE. As such, the local pfADA diagnostic cut-off value is set at $26.5 \mathrm{U} / \mathrm{L}$, with a sensitivity of $87.3 \%$, specificity of $93.2 \%$, PPV of $79.2 \%$, NPV of $96.1 \%$, and accuracy of $91.9 \%$.

Implications for clinical practice or policy

Among patients with pfADA level of $\leq 100 \mathrm{U} / \mathrm{L}$, when pfADA level is $\geq 26.5 \mathrm{U} / \mathrm{L}$ with early negative findings in pleural fluid for malignancy and non-tuberculous infection, it is probably appropriate to manage the patient as a case of TBPE, without additionally performing pleural biopsy (also a surrogate marker for TBPE), but remain vigilant for a $20.8 \%$ (1 minus PPV) chance of mistaking non-tuberculous diseases as TBPE.

- When pfADA level is $<26.5 \mathrm{U} / \mathrm{L}$ with early negative findings in pleural fluid for malignancy and non-tuberculous infection, tuberculosis is highly unlikely, but caution should be exercised because of a $3.9 \%$ (1 minus NPV) chance of mistaking TBPE for another disease.

- Other investigations are always indicated when the clinical progress is incompatible with the working diagnosis. 


\section{Introduction}

Adenosine deaminase (ADA) is an enzyme involved in purine metabolism, with its primary function in the development and maintenance of the immune system. There are at least two ADA isoforms: ADA1 and ADA2. Whereas ADA1 is found in most body cells (especially lymphocytes and macrophages), ADA2 is predominantly found in the human plasma and serum, and co-exists with ADA1 in macrophages. Absence of ADA1 causes severe combined immunodeficiency. Serum ADA2 level is increased in collagen vascular disease, ${ }^{1,2}$ and most cancers.

Many studies have suggested that pleural fluid adenosine deaminase (pfADA) is useful in the diagnosis of tuberculous pleural effusion (TBPE). ${ }^{3-13}$ The merits of using pfADA include its low cost, short turnaround time, and high sensitivity and specificity. ${ }^{3,12}$ Notwithstanding possibly better sensitivity and specificity for detecting TBPE by combining ADA1 or ADA2 in pleural fluid (PF) with other PF biomarkers such as tumour necrosis factor-alpha, interleukin 27, interferon-gamma and dipeptidyl peptidase $I V,{ }^{14-17}$ it may not be cost-effective to combine pfADA with other PF biomarkers. ${ }^{18}$ Although ADA2 is predominantly increased in TBPE, and ADA1 is more commonly associated with pleural effusion due to pyogenic bacteria, ${ }^{19}$ determination of ADA1 and ADA2 may not provide a diagnostic advantage over the use of total pfADA. ${ }^{20}$

A standardised and automated method (Diazyme commercial kit; Diazyme Laboratories, San Diego [CA], United States) has been developed to determine pfADA activity. The test performance of pfADA has largely been evaluated by including all cases with pleural effusion, and estimating its sensitivity and specificity with reference to an optimal cut-off value. Some studies fine-tuned the test performance by restricting the analysis to subjects with lymphocytic exudates ${ }^{9,13}$ or to young adults. ${ }^{21}$ In Hong Kong, pfADA has been measured centrally by the Chemical Pathology Laboratory at the Princess Margaret Hospital using the Diazyme commercial kit. In the absence of a diagnostic cut-off value established from local data, pfADA level of $\geq 30 \mathrm{U} / \mathrm{L}$ has been used territory-wide in Hong Kong for detecting TBPE. This is with reference to a retrospective Thai study of 59 (33.1\%) patients with TBPE among 178 patients with predominantly exudative lymphocytic pleural effusion. ${ }^{22}$ It suggested a sensitivity of $82 \%$ and specificity of $91 \%$ for pfADA level of $\geq 30 \mathrm{U} / \mathrm{L}$, as measured by the Diazyme commercial kit. ${ }^{22}$ Corresponding estimates of positive predictive value (PPV) and negative predictive value (NPV) were $81.4 \%$ and $90.8 \%$, respectively. ${ }^{22}$

Although pfADA rapidly detects TBPE, it is often assessed alongside other tests that include sputum bacteriology for acid-fast bacilli (AFB) and

\section{在香港成人病例中，以腺苷脫氨酶水平作診斷結 核性胸腔積液的優化研究}

鄭國釗、陳銘釗、梁偉民、江福葉、麥苗、陳栢林、余衛祖

引言：胸腔積液腺苷脱氨酶（pfADA）水平可用於快速診斷結核性胸 腔積液。本文旨在估算pfADA在本地用作診斷結核性胸腔積液的截取 值, 並探討如何優化其效用。

方法：本研究回顧香港一組公立醫院內的病歷紀錄, 分析 2011 年 1 月 1 日至 12 月 31 日期間, 用Diazyme測試劑檢測的pfADA水平。分析中 考慮各種不同情景, 包括有否使用Light的標準去篩選, 以及有沒有考 慮胸水和胸膜活檢的早期（2週內）結果。對於每種情景, 我們使用 ROC曲線來找出pfADA的截取值, 並估計其陽性和陰性預測值。

結果：可供研究的病歷紀錄有 860 份。胸腔積液的病因包括：充血 性心臟衰竭、慢性腎功能衰竭、或肝腎疾病導致的低蛋白血症共 246例（28.6\%）；惡性腫瘤198例（23.0\%）；非結核性感染168例 （19.5\%）；結核性胸腔積液157例（18.3\%）；以及其他原因91例 $(10.6 \%)$ 。所有結核性胸腔積液的pfADA水平均為 $\leq 100 \mathrm{U} / \mathrm{L}$ 。當分 析僅限於pfADA水平為 $\leq 100 \mathrm{U} / \mathrm{L} 及$ 早期胸水中沒有惡性腫瘤和非結 核性感染的689例時, 陽性預測值顯著增加, 但陰性預測值未明顯降 低。使用同樣方法, 即使額外使用Light的標準篩檢, 或附加考慮封閉 的胸膜活檢, 都不能進一步提高預測值。因此, 我們估算pfADA的診 斷截取值為 $26.5 \mathrm{U} / \mathrm{L}$, 其敏感性為 $87.3 \%$, 特異性 $93.2 \%$, 陽性預測值 $79.2 \%$, 陰性預測值 $96.1 \%$, 準確性 $91.9 \%$ 。性別、年齡和共病並沒有 明顯影響結核性胸腔積液的預測值。

結論：在母須使用Light的標準篩選，僅把分析限制在pfADA水平為 $\leq 100 \mathrm{U} / \mathrm{L} 及$ 考慮早期胸水結果的情況下, 我們找到pfADA的本地診斷 截取值

other pathogens, sputum cytology, PF bacteriology for AFB and other pathogens, PF biochemistry, PF cytology, and pleural biopsy. Restricting analysis to patients with exudative pleural effusion may help optimise the utility of pfADA for detecting TBPE. Excluding patients with an early diagnosis of nontuberculous disease, notably malignancy and nontuberculous infection, may also help improve the utility of pfADA for TBPE. 5,9,23-26

Diagnostic test accuracy depends on sensitivity and specificity, which are relatively stable, and pre-test probability that can be enhanced by selecting appropriate patients. In this study, we aimed to optimise its utility by increasing the pretest probability, and establish a local diagnostic pfADA cut-off value for adult TBPE. Additionally, we evaluated whether the prediction of TBPE using the pfADA cut-off value was affected by sex, age, or co-morbidity.

\section{Methods}

We retrospectively searched a centralised computerised database for consecutive PF specimens tested for ADA from 1 January 2011 to 31 December 2011 and assembled a cohort of patients with exudative pleural effusion. These patients were all 
managed at a cluster of public hospitals that served a large population in western Kowloon of Hong Kong. At least $90 \%$ of patients with pleural effusion had PF tested for ADA. We considered pfADA alongside early (within 2 weeks) findings in PF and pleural biopsy, with and without applying Light's criteria ${ }^{27}$ in multiple scenarios. For each scenario, we used the receiver operating characteristic (ROC) curve and the Youden Index (the point of maximal summation of sensitivity and specificity estimates) to identify an optimal pfADA diagnostic cut-off value for TBPE, and estimated the corresponding PPV and NPV. The Youden Index maximises the difference between the true-positive rate (sensitivity) and the false-positive rate ( 1 minus specificity), thereby maximising the correct classification rate. When the Youden Index comprised more than 1 point, we also considered the point at minimal distance between the ROC curve and the coordinate with $100 \%$ specificity and $100 \%$ sensitivity.

The following data were collected by review of medical records that had been created and maintained by clinicians who were unaware of the study hypothesis: sex, age (at the time of initial diagnosis), smoking history, drinking history, comorbidity (chronic obstructive pulmonary disease, diabetes mellitus, chronic renal failure), use of immunosuppressive treatment for at least 1 month in the past year, nature of PF (exudate vs transudate by Light's criteria), sputum AFB smear and culture, PF AFB smear and culture, PF bacterial and fungal stain, PF culture of other bacteria or fungus, pleural biopsy findings, other significant findings related to initial or definitive diagnosis, the early diagnosis (within 2 weeks after checking pfADA), and the definitive diagnosis (by 1 year after checking pfADA).

This study was conducted in accordance with the amended Declaration of Helsinki, and approved by the Kowloon West Cluster Research Ethics Committee (IRB approval number: KW/EX-13139(69-17)) and the Department of Health Ethics Committee (IRB approval number: L/M 400/2013).

\section{Definitions}

The exudative versus transudative nature of PF was established by reference to Light's criteria that classify PF as exudative in the presence of any one of the following: ratio of protein in PF to serum $>0.5$, ratio of lactate dehydrogenase (LDH) in PF to serum $>0.6$, and PF LDH level of $>200 \mathrm{IU} / \mathrm{L} .{ }^{27}$

A definitive diagnosis of TBPE was made when Mycobacterium tuberculosis complex was isolated in culture of PF or parietal pleura, or any one of the following in the absence of an alternative diagnosis by 1 year after pfADA checking: (i) granulomatous inflammation of parietal pleura, (ii) culture-proven pulmonary tuberculosis (TB) with pleural effusion and compatible response to TB treatment, (iii) a clinical diagnosis of TBPE with compatible response to TB treatment, or (iv) AFB and/or positive findings from nucleic acid amplification tests in PF or parietal pleura. An early diagnosis of TBPE was made when pleural biopsy showed granulomatous inflammation in the absence of an alternative cause, or rarely, the presence of AFB or positive findings from nucleic acid amplification tests in PF or parietal pleura.

Parapneumonic effusion refers to any pleural effusion secondary to pneumonia or lung abscess. ${ }^{28}$ The PF is often exudative with a predominance of neutrophils. ${ }^{28}$ It can be 'simple' (with sterile exudate) or 'complicated' (with progression to a fibrinopurulent state), characterised by $\mathrm{pH}<7.2$, glucose level of $<2.2$ $\mathrm{mmol} / \mathrm{L}$, and LDH level of $>1000 \mathrm{IU} / \mathrm{L} .{ }^{29}$ Empyema thoracis is a complicated parapneumonic effusion with frank pus. ${ }^{28}$ A definitive diagnosis of simple non-tuberculous parapneumonic effusion was made if the PF was exudative and sterile with LDH level of $\leq 1000 \mathrm{IU} / \mathrm{L}$ and if there was a compatible clinical response to empirical antibiotic treatment, in the absence of an alternative diagnosis by 1 year after the first attempt of diagnostic thoracentesis. Without an identifiable non-tuberculous pathogen, we considered it impossible to confidently make an early diagnosis of simple non-tuberculous parapneumonic effusion. A definitive diagnosis of complicated nontuberculous parapneumonic effusion, or empyema thoracis in the presence of frank pus or compatible radiological signs on chest computed tomographic scan was made if the PF was exudative with a nontuberculous pathogen (demonstrated by positive stain/culture) in PF or parietal pleura, or LDH level of $>1000 \mathrm{IU} / \mathrm{L}$, and compatible clinical response to empirical antibiotic treatment and/or drainage, in the absence of an alternative diagnosis by 1 year after the first attempt of diagnostic thoracentesis. An early diagnosis of complicated non-tuberculous parapneumonic effusion, or empyema thoracis in the presence of frank pus or compatible radiological signs, was made when a non-tuberculous pathogen could be identified in PF or parietal pleura.

Malignant pleural effusion refers to the presence of malignant cells in PF and/or parietal pleura. ${ }^{30} \mathrm{~A}$ definitive diagnosis of malignant pleural effusion was made if malignant cells were found in PF and/ or parietal pleura, or clinical/radiological findings were compatible with malignant pleural effusion in the absence of an alternative diagnosis by 1 year after the first attempt of diagnostic thoracentesis. An early diagnosis of malignant pleural effusion was made when malignant cells could be demonstrated in PF or parietal pleura.

\section{Statistical analysis}

Chi squared test (for categorical data), Fisher's exact test (for categorical data), McNemar's test (for paired data), Student's $t$ test (for continuous variables 
normally distributed), and Mann-Whitney $U$ test (for continuous variables not normally distributed) were used as appropriate to evaluate the association between TBPE and the pfADA cut-off as well as demographic factors and co-morbidity. Factors with a $P$ value of $<0.25$ by univariate analysis were forced into a logistic regression model after considering multicollinearity.

\section{Laboratory methods}

Throughout the study period, ADA activity was measured by the same automated method, the Diazyme commercial kit in the Beckman Coulter UniCel DxC 800 Synchron Clinical System. The automated Diazyme method has been validated. ${ }^{31}$

\section{Results}

Search from the computerised database of ADA assay from 1 January to 31 December 2011 identified a total of 903 independent PF specimens from 903 patients. We evaluated 860 patients with pleural effusion and pfADA after excluding 42 cases that were peritoneal rather than $\mathrm{PF}$ and one case with no medical record. Table 1 shows their definitive diagnoses and the corresponding pfADA value. Pleural effusion was caused by congestive heart failure, chronic renal failure, hypoalbuminaemia, or nephrotic syndrome/nephropathy with membranous glomerulonephritis in 246 (28.6\%) cases, malignancy in $198(23.0 \%)$, non-tuberculous infection (simple non-tuberculous parapneumonic effusion, complicated non-tuberculous parapneumonic effusion other than empyema, and non-tuberculous empyema thoracis) in 168 (19.5\%), TBPE in 157 (18.3\%), and miscellaneous or unknown causes in 91 (10.6\%). By Light's criteria, 626 (72.8\%) cases were classified as exudative, $222(25.8 \%)$ as transudative, and $12(1.4 \%)$ as indeterminate (lack of data).

Among the 198 patients with malignant pleural effusion, an early diagnosis could be established by detecting malignant cells in PF in 136 (68.7\%), including 21 also detected by pleural biopsy (20 closed and 1 open), and seven (3.5\%) by pleural biopsy alone ( 5 closed and 2 open). Malignant pleural effusion was caused by lung cancer in 152 (76.8\%) patients, lymphoid or haematological malignancy in $12(6.1 \%)$, unknown primary in nine (4.5\%), gastric cancer in five (2.5\%), ovarian cancer in four $(2.0 \%)$, breast cancer in four (2.0\%), liver cancer in two $(1.0 \%)$, pancreatic cancer in two $(1.0 \%)$, and one $(0.5 \%)$ each by cancer in the nasopharynx, tongue, oesophagus, unspecified gastrointestinal tract, kidney, urinary bladder, prostate, and nerve.

Among the 168 patients with non-tuberculous infection, infection was bacteriologically confirmed by PF culture in 21 (12.5\%) cases with nontuberculous empyema thoracis (including 19 with early diagnosis), and 10 (6.0\%) with complicated non-tuberculous parapneumonic effusion (including 9 with early diagnosis).

Among the 157 patients with TBPE, the diagnosis was (1) bacteriologically confirmed by PF culture in $62(39.5 \%)$ including four also confirmed

TABLE I. Definitive diagnosis of pleural effusion among a cohort of 860 patients with pfADA level measured

\begin{tabular}{|c|c|c|}
\hline Diagnosis & No. $(\%)$ of patients & Median (range) pfADA (U/L)* \\
\hline Malignant pleural effusion & $198(23.0)$ & $9.7(<2.4$ to 245$)$ \\
\hline Tuberculous pleural effusion & $157(18.3)$ & $44(<2.4$ to 86$)$ \\
\hline Congestive heart failure $†$ & $139(16.2)$ & $4.1(<2.4$ to 25$)$ \\
\hline Simple non-tuberculous parapneumonic effusion & $97(11.3)$ & $11(<2.4$ to 52$)$ \\
\hline Uncertain diagnosis & $72(8.4)$ & $9.4(<2.4$ to 104$)$ \\
\hline Chronic renal failure or uraemic pleuritis $\ddagger$ & $59(6.9)$ & $4.6(<2.4$ to 30$)$ \\
\hline Non-tuberculous empyema thoracis & $42(4.9)$ & 48 (8.9 to 343$)$ \\
\hline Cirrhosis with ascites or hypoalbuminaemia other than cirrhosis§ & $42(4.9)$ & $4.6(<2.4$ to 13$)$ \\
\hline Complicated non-tuberculous parapneumonic effusion other than empyema & $29(3.4)$ & 25 (2.4 to 79$)$ \\
\hline Liver abscess or parapharyngeal abscess or postoperative subphrenic collection & $8(0.9)$ & 11 (9.5 to 180$)$ \\
\hline Nephrotic syndrome or nephropathy with membranous glomerulonephritis & $6(0.7)$ & $3.2(<2.4$ to 8.9$)$ \\
\hline Pneumothorax or haemothorax or haemopneumothorax & $5(0.6)$ & 12 (3.9 to 22$)$ \\
\hline Connective tissue disorder or serositis & $3(0.3)$ & 21 (8.1 to 23$)$ \\
\hline Meigs syndrome & $2(0.2)$ & 4.6 (4.4 to 4.8$)$ \\
\hline Pleuroperitoneal fistula & $1(0.1)$ & $<2.4$ \\
\hline
\end{tabular}

Abbreviation: pfADA = pleural fluid adenosine deaminase

* Readings of $<2.4 \mathrm{U} / \mathrm{L}$ could not be accurately quantified

+ 14 Patients had chronic renal failure

$\ddagger 4$ Patients had end-stage renal failure on chronic ambulatory peritoneal dialysis

$\S 2$ Patients had hepatocellular carcinoma, 2 had congestive heart failure, and 2 had chronic renal failure 
by pleural tissue culture and 26 also suggested by pleural biopsy; (2) bacteriologically confirmed by pleural tissue culture in $12(7.6 \%)$ including four also confirmed by PF culture and nine also suggested by pleural biopsy; (3) histologically suggested by pleural biopsy in 74 ( 69 closed and 5 open; $47.1 \%$ ) including 26 also confirmed by PF culture and nine also confirmed by pleural tissue culture; (4) clinically suggested by pulmonary TB in $44(28.0 \%)$ including 26 solely by clinical correlation with radiological progress; and (5) clinically suggested by culture-proven TB ascites in one (0.6\%). Sputum AFB smear was positive in nine (5.7\%) patients, with $M$ tuberculosis complex isolated in the sputum culture of 51 (32.5\%). An early diagnosis could be established in 65 (41.4\%), using pleural biopsy in 64 and polymerase chain reaction in PF in one. The majority $(n=152)$ of patients with TBPE were Chinese.

Among 90 patients with TBPE and closed pleural biopsy performed, TBPE was detected by pleural biopsy in $69(76.7 \%)$ and pfADA cut-off level in 85 (94.4\%). The difference was statistically significant $(\mathrm{P}<0.005$ by McNemar's test).

Table 2 shows the distribution of pfADA levels stratified by the nature of PF and tuberculous versus non-tuberculous pleural effusion. The prevalence (pre-test probability) of TBPE was significantly higher among exudative $(24.3 \%)$ than transudative $(2.3 \%)$ cases. All cases with TBPE had pfADA level of $\leq 86$ $\mathrm{U} / \mathrm{L}$. With pfADA level of $>86 \mathrm{U} / \mathrm{L}$, all cases $(\mathrm{n}=18)$ with exudative non-tuberculous pleural effusion had pfADA level of $>100 \mathrm{U} / \mathrm{L}$ of whom 13 patients had non-tuberculous empyema thoracis, two had lymphoma, one had plasmacytoma, one had liver abscess, and one had an uncertain diagnosis.

Figure 1 shows how we proceeded to increase the pre-test probability of TBPE by first excluding transudates, and then stepwise excluding nontuberculous patients to further increase the pre-test probability. For each scenario, the pfADA cut-off value was tabulated alongside estimates of sensitivity, specificity, PPV, and NPV. Restricting analysis to 461 patients with exudative pleural effusion, pfADA level of $\leq 100 \mathrm{U} / \mathrm{L}$, and early negative findings for non-tuberculous infection and malignancy in PF significantly increased PPV from $66.3 \%$ to $79.5 \%$ and non-significantly reduced NPV from $97.1 \%$ to $94.5 \%$. Further excluding seven patients with an early diagnosis of malignancy by pleural biopsy resulted in no change to PPV and a non-significant decrease in NPV. Figure 2 shows an alternative approach that disregards Light's criteria. Restricting analysis to 689 patients with pfADA level of $\leq 100 \mathrm{U} / \mathrm{L}$ and early negative findings for non-tuberculous infection and malignancy in PF also significantly increased PPV from $66.3 \%$ to $79.2 \%$ and non-significantly reduced NPV from $97.1 \%$ to $96.1 \%$. Further excluding seven patients with an early diagnosis of malignancy by pleural biopsy resulted in no change to PPV and a non-significant decrease in NPV from $96.12 \%$ to $96.07 \%$. With no significant difference in PPV $(\mathrm{P}=0.938)$ or NPV $(\mathrm{P}=0.279)$ between the two approaches, the utility of pfADA may be optimised by applying a diagnostic cut-off among patients with pfADA level of $\leq 100 \mathrm{U} / \mathrm{L}$ and early negative findings for malignancy and non-tuberculous infection in PF, without considering Light's criteria or pleural biopsy. As such, pfADA level of $\geq 26.5 \mathrm{U} / \mathrm{L}$, ascertained from the ROC curve using the Youden Index, detected TBPE with a sensitivity of $87.3 \%$, specificity of $93.2 \%$, PPV of $79.2 \%$, NPV of $96.1 \%$, and accuracy of $91.9 \%$ (Fig 3).

Table 3 shows different causes of pleural effusion above and below the diagnostic pfADA cut-off value of $26.5 \mathrm{U} / \mathrm{L}$ among the 689 patients with pfADA level of $\leq 100 \mathrm{U} / \mathrm{L}$, and early negative findings for malignancy and non-tuberculous infection in PF. It is noteworthy that in the seven (4.0\%) patients with pfADA level of $\geq 26.5 \mathrm{U} / \mathrm{L}$ and $64(12.4 \%)$ patients with pfADA level of $<26.5 \mathrm{U} / \mathrm{L}$, the diagnosis was uncertain. Among 157 patients with TBPE, 137 (87.3\%, sensitivity) tested positive (pfADA $\geq 26.5 \mathrm{U} / \mathrm{L}$ ), with false-negative results in 20 (12.7\%, the false-negative rate or 1 minus sensitivity). Among 532 patients with non-tuberculous pleural effusion, 496 (93.2\%, specificity) tested negative (pfADA $<26.5 \mathrm{U} / \mathrm{L}$ ), with false-positive results in 36 (6.8\%, false-positive rate or 1 minus specificity).

TABLE 2. Distribution of pfADA levels stratified by the nature of pleural fluid and tuberculous versus non-tuberculous pleural effusion

\begin{tabular}{|c|c|c|c|c|c|}
\hline \multirow{2}{*}{$\begin{array}{l}\text { Nature of pleural fluid by } \\
\text { Light's criteria }\end{array}$} & \multicolumn{2}{|c|}{ Non-tuberculous pleural effusion } & \multicolumn{2}{|c|}{ Tuberculous pleural effusion } & \multirow[t]{2}{*}{ Total } \\
\hline & No. of patients & $\begin{array}{c}\text { Median (range) pfADA } \\
(\mathrm{U} / \mathrm{L})^{\star}\end{array}$ & No. of patients & $\begin{array}{c}\text { Median (range) pfADA } \\
(\mathrm{U} / \mathrm{L})^{\star}\end{array}$ & \\
\hline Exudate & 474 & $11.0(<2.4$ to 343$) \dagger$ & 152 & 44.0 (5.3 to 86.0$)$ & 626 \\
\hline Transudate & 217 & $4.0(<2.4$ to 13$)$ & 5 & $13.0(<2.4$ to 45$)$ & 222 \\
\hline Indeterminate (lack of data)‡ & 12 & $8.9(<2.4$ to 300$)$ & 0 & Not applicable & 12 \\
\hline
\end{tabular}

Abbreviation: pfADA = pleural fluid adenosine deaminase

* Readings of $<2.4 \mathrm{U} / \mathrm{L}$ could not be accurately quantified

+ For those with non-tuberculous pleural effusion and pfADA >86 U/L, all had pfADA >100 U/L

‡ Diagnoses included malignant pleural effusion in 7, non-tuberculous empyema thoracis in I, pneumothorax in I, simple non-tuberculous parapneumonic effusion in 2, and hypoalbuminaemia due to causes other than cirrhosis in I 


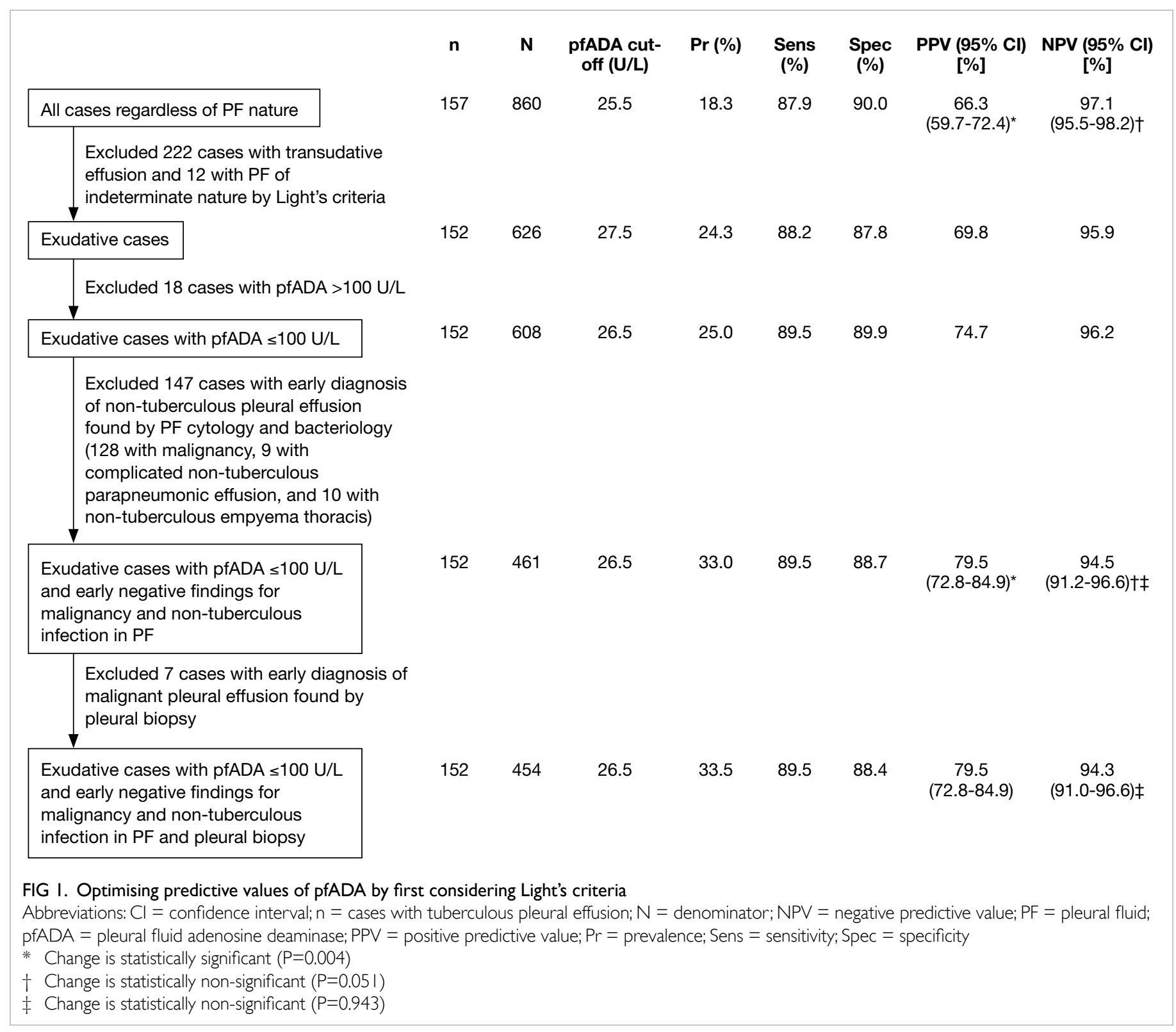

Among 173 patients who tested positive, 137 (79.2\%, PPV) were true-positive, and 36 (20.8\%, 1 minus $\mathrm{PPV}$ ) were false-positive with non-tuberculous diseases mistaken for TBPE: 10 with complicated non-tuberculous parapneumonic effusion, 10 with non-tuberculous empyema thoracis, seven with uncertain diagnosis, four with malignant pleural effusion, four with simple non-TB parapneumonic effusion, and one with chronic renal failure. Among 516 patients tested negative, 496 (96.1\%, NPV) were true-negative, and 20 (3.9\%, 1 minus NPV) were falsenegative with TBPE mistaken for non-tuberculous pleural effusion. Among 689 test results, 633 (91.9\%) were accurate and comprised 137 true-positive and 496 true-negative results.

A logistic regression model that considered sex, age, and co-morbidity alongside the pfADA diagnostic cut-off value identified pfADA level of $\geq 26.5 \mathrm{U} / \mathrm{L}$ as the only significant predictive variable of TBPE (Table 4).

\section{Discussion}

As a limited surrogate marker for TBPE, pfADA level must be interpreted alongside other clinical, radiological, and laboratory findings that help exclude non-tuberculous diseases, thereby increasing the pre-test probability of TBPE and the PPV. Using local data as measured by the Diazyme commercial kit, we demonstrated that TBPE was unlikely when pfADA level was $>100 \mathrm{U} / \mathrm{L}$. Restricting analysis to patients with pfADA level of $\leq 100 \mathrm{U} / \mathrm{L}$ and early (within 2 weeks) negative findings for malignancy and nontuberculous infection in PF significantly increased the PPV and non-significantly reduced the NPV. Using this approach, neither additionally restricting 


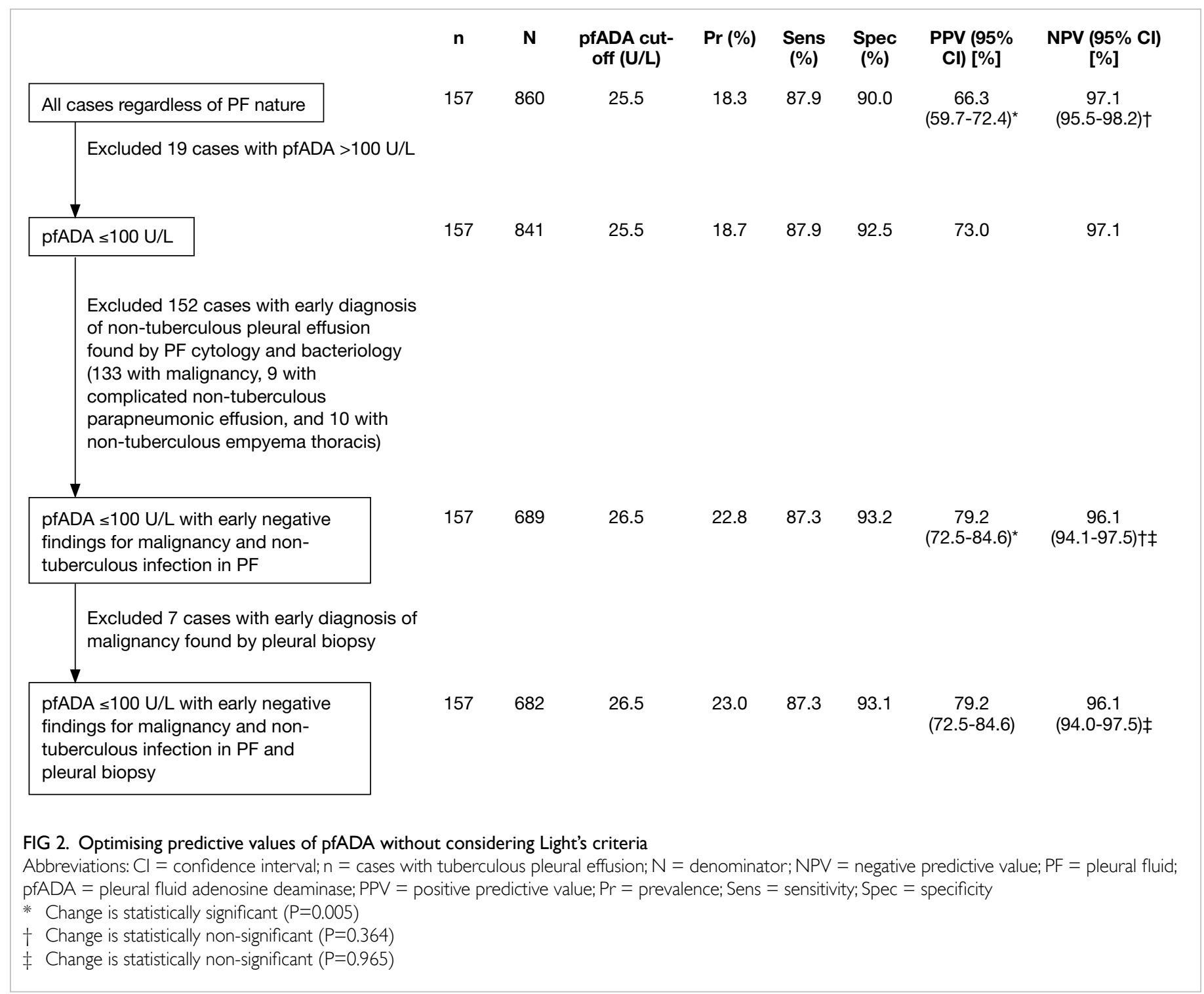

analysis to exudates by Light's criteria, nor adding closed pleural biopsy, would further enhance the predictive value of pfADA for TBPE. This might be explained by the fact that pfADA level of $>13 \mathrm{U} / \mathrm{L}$ excluded all non-tuberculous transudative cases (Table 2), and that pfADA was significantly more sensitive than closed pleural biopsy for TBPE. A recent study, which demonstrated a need to suspect empyema or lymphoma when the pfADA level was extremely high, ${ }^{32}$ corroborated our findings regarding the low likelihood of TBPE when pfADA level was $>100 \mathrm{U} / \mathrm{L}$. Furthermore, we demonstrated that the prediction of TBPE using the pfADA diagnostic cut-off value was not affected by sex, age, or co-morbidity. Another study that developed a predictive model for TBPE also failed to show any significant association between TBPE and either age or sex..$^{33}$

Among patients with pfADA level of $\leq 100 \mathrm{U} / \mathrm{L}$, when pfADA level is $\geq 26.5 \mathrm{U} / \mathrm{L}$ with early negative findings in PF for malignancy and non-tuberculous infection, it is probably appropriate to manage the patient as a case of TBPE, without additionally performing pleural biopsy (also a surrogate marker for TBPE). Nonetheless, it is important to remain vigilant due to a $20.8 \%$ (1 minus PPV) chance of mistaking non-tuberculous diseases for TBPE and prescribing unnecessary $\mathrm{TB}$ treatment. When pfADA level is $<26.5 \mathrm{U} / \mathrm{L}$ with early negative findings in PF for malignancy and non-tuberculous infection, TB is highly unlikely. Again caution should be exercised in the presence of a $3.9 \%$ ( 1 minus NPV) chance of mistaking TBPE for other diseases. Tuberculosis is potentially fatal although effective treatment can reduce morbidity and mortality. Yet standard TB treatment is not without harmful sideeffects that include hepatotoxicity. This occurs in $1 \%$ to $3 \%$ of patients on average and becomes more prevalent among the elderly people and those with underlying liver disease. ${ }^{34,35}$ Additionally, treating 
non-tuberculous disease as TB may also delay the diagnosis of other diseases including malignancy. It is important to balance the benefits of TB treatment against the risks when using a pfADA cut-off value to diagnose TBPE. In general, if the test suggests TBPE, and the risk of morbidity or mortality from untreated TB is substantial, it is prudent to promptly start TB treatment, and closely monitor treatment progress, with further investigations for other diseases conducted concurrently or as soon as treatment response is considered suboptimal. Other investigations are always indicated when the clinical progress is incompatible with the working diagnosis.

A major drawback of this study was its retrospective nature and related selection and misclassification bias. Selection bias may be modest as public hospitals provide approximately $90 \%$ of hospital care in Hong Kong, and we included every consecutive and non-duplicated PF sample from all patients managed during the study period in a large public hospital cluster in which at least $90 \%$ patients with pleural effusion had PF tested for ADA. Misclassification bias may occur. Efforts made by clinicians to confirm TB disease may be selectively affected by knowledge about the association between pfADA and TB. Non-tuberculous infection could have been misclassified as TB, thereby overestimating

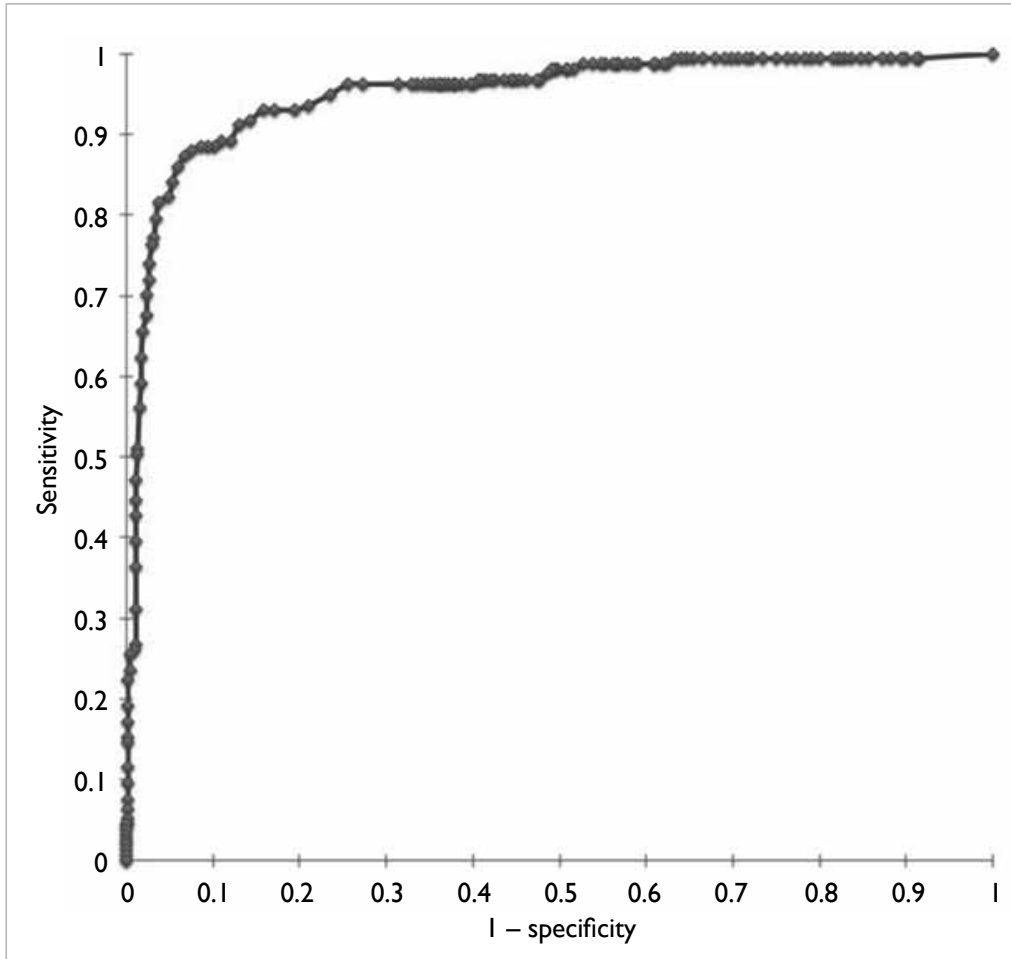

FIG 3. Receiver operating characteristic curve based on data of 689 patients with pleural fluid adenosine deaminase level of $\leq 100 \mathrm{U} / \mathrm{L}$ and early (within 2 weeks) negative findings for malignancy and non-tuberculous infection in pleural fluid

TABLE 3. Different causes of pleural effusion above and below the diagnostic pfADA cut-off value among 689 patients with pfADA level of $\leq 100 \mathrm{U} / \mathrm{L}$, and early (within 2 weeks) negative findings for malignancy and non-tuberculous infection in pleural fluid

\begin{tabular}{|c|c|c|}
\hline \multirow[t]{2}{*}{ Cause of pleural effusion } & \multicolumn{2}{|c|}{ No. (\%) of patients } \\
\hline & pfADA $<26.5$ U/L $(n=516)$ & pfADA $\geq 26.5 \mathrm{U} / \mathrm{L}(\mathrm{n}=173)$ \\
\hline TBPE & $20(3.9)$ & $137(79.2)$ \\
\hline Complicated non-tuberculous parapneumonic effusion & $10(1.9)$ & $10(5.8)$ \\
\hline Non-tuberculous empyema thoracis & $9(1.7)$ & $10(5.8)$ \\
\hline Uncertain diagnosis & $64(12.4)$ & $7(4.0)$ \\
\hline Malignant pleural effusion & $57(11.0)$ & $4(2.3)$ \\
\hline Simple non-tuberculous parapneumonic effusion & $93(18.0)$ & $4(2.3)$ \\
\hline Chronic renal failure or uraemic pleuritis & $58(11.2)$ & $1(0.6)$ \\
\hline Congestive heart failure & $139(26.9)$ & 0 \\
\hline Cirrhosis with ascites or hypoalbuminaemia due to other causes & $42(8.1)$ & 0 \\
\hline $\begin{array}{l}\text { Liver abscess or parapharyngeal abscess or postoperative } \\
\text { subphrenic collection }\end{array}$ & $7(1.4)$ & 0 \\
\hline Pneumothorax or haemothorax or haemopneumothorax & $5(1.0)$ & 0 \\
\hline Connective tissue disorder or serositis & $3(0.6)$ & 0 \\
\hline Meigs syndrome & $2(0.4)$ & 0 \\
\hline Nephrotic syndrome or nephropathy & $6(1.2)$ & 0 \\
\hline Pleuroperitoneal fistula & $1(0.2)$ & 0 \\
\hline
\end{tabular}

Abbreviations: pfADA = pleural fluid adenosine deaminase; TBPE = tuberculous pleural effusion 
TABLE 4. Univariate and multiple logistic regression analyses of tuberculous versus non-tuberculous exudative pleural effusion among $46 \mathrm{I}$ patients with exudative pleural effusion, pfADA level of $\leq 100 \mathrm{U} / \mathrm{L}$, and early (within 2 weeks) negative findings for malignancy and non-tuberculous infection in pleural fluid

\begin{tabular}{|c|c|c|c|c|}
\hline \multirow[t]{2}{*}{ Variable } & \multicolumn{3}{|c|}{ Univariate analysis, No. (\%) of patients* } & \multirow{2}{*}{$\begin{array}{c}\text { Multiple logistic } \\
\text { regression analysis† } \\
\text { Adjusted OR }(95 \% \mathrm{Cl})\end{array}$} \\
\hline & Non-tuberculous $(n=309)$ & Tuberculous $(n=152)$ & $P$ value & \\
\hline \multicolumn{5}{|l|}{ Sex } \\
\hline Male & $190(61.5)$ & $109(71.7)$ & 0.03 & $0.7(0.3-1.5)$ \\
\hline Female & $119(38.5)$ & $43(28.3)$ & & \\
\hline Median (IQR) age upon enrolment (years) & $74(58-83)$ & $65(43-79)$ & $<0.001$ & $0.99(0.98-1.01)$ \\
\hline \multicolumn{5}{|l|}{$\operatorname{pfADA} \geq 26.5 \mathrm{U} / \mathrm{L}$} \\
\hline No & $274(88.7)$ & $16(10.5)$ & $<0.001$ & $59.8(31.7-112.9)$ \\
\hline Yes & $35(11.3)$ & $136(89.5)$ & & \\
\hline \multicolumn{5}{|l|}{ Smoking } \\
\hline No history & $204(66.0)$ & $86(56.6)$ & 0.05 & $1.1(0.5-2.1)$ \\
\hline Current smoker or ex-smoker & $105(34.0)$ & $66(43.4)$ & & \\
\hline \multicolumn{5}{|l|}{ Drinking } \\
\hline No history & $267(86.4)$ & $127(83.6)$ & 0.41 & - \\
\hline Habitual drinker, ex-drinker, or social drinker & $42(13.6)$ & $25(16.4)$ & & \\
\hline \multicolumn{5}{|l|}{ Chronic obstructive pulmonary disease } \\
\hline No & $279(90.3)$ & $142(93.4)$ & 0.26 & - \\
\hline Yes & $30(9.7)$ & $10(6.6)$ & & \\
\hline \multicolumn{5}{|l|}{ Diabetes mellitus } \\
\hline No & $230(74.4)$ & $130(85.5)$ & 0.01 & $0.8(0.4-1.9)$ \\
\hline Yes & $79(25.6)$ & $22(14.5)$ & & \\
\hline \multicolumn{5}{|l|}{ Chronic renal failure } \\
\hline No & $245(79.3)$ & $132(86.8)$ & 0.05 & $0.8(0.3-1.8)$ \\
\hline Yes & $64(20.7)$ & 20 (13.2) & & \\
\hline \multicolumn{5}{|l|}{ Immunosuppressive treatment } \\
\hline No & $302(97.7)$ & $149(98.0)$ & 1.0 & - \\
\hline Yes & $7(2.3)$ & $3(2.0)$ & & \\
\hline
\end{tabular}

Abbreviations: $\mathrm{Cl}=$ confidence interval; $I Q R$ = interquartile range; $O R=$ odds ratio; pfADA = pleural fluid adenosine deaminase

* Unless stated otherwise

+ Variables with $\mathrm{P}<0.25$ by univariate analysis were forced in during multiple logistic regression analysis

PPV or underestimating NPV. On the other hand, TBPE could also have been misclassified as non-TB, thereby underestimating PPV or overestimating NPV. Another possible source of misclassification bias was uncertain diagnosis (Table 3), which was considered as non-tuberculous during analysis. Of note, TBPE labelled as uncertain diagnosis could have caused an underestimation of PPV or overestimation of NPV. Nonetheless, the lack of a definitive diagnosis by 1 year might suggest a low likelihood of TBPE, thereby reducing the impact of this misclassification bias.

We have established a pfADA diagnostic cut-off value for TBPE by restricting analysis to patients with pfADA level of $\leq 100 \mathrm{U} / \mathrm{L}$, and considering early PF findings for malignancy and non-tuberculous infection, but not Light's criteria.

\section{Declaration}

All authors have disclosed no conflicts of interest.

\section{References}

1. Sari RA, Taysi S, Yilmaz O, Bakan N. Correlation of serum levels of adenosine deaminase activity and its isoenzymes with disease activity in rheumatoid arthritis. Clin Exp Rheumatol 2003;21:87-90.

2. Stancíková M, Lukác J, Istok R, Cristalli G, Rovensky J. Serum adenosine deaminase activity and its isoenzyme pattern in patients with systemic lupus erythematosus. Clin Exp Rheumatol 1998;16:583-6.

3. Gupta DK, Suri JC, Goel A. Efficacy of adenosine deaminase in the diagnosis of pleural effusions. Indian J Chest Dis Allied Sci 1990;32:205-8.

4. Bañales JL, Pineda PR, Fitzgerald JM, Rubio H, Selman M, 
Salazar-Lezama M. Adenosine deaminase in the diagnosis of tuberculous pleural effusions. A report of 218 patients and review of the literature. Chest 1991;99:355-7.

5. Valdés L, Alvarez D, San José E, et al. Value of adenosine deaminase in the diagnosis of tuberculous pleural effusions in young patients in a region of high prevalence of tuberculosis. Thorax 1995;50:600-3.

6. Burgess LJ, Maritz FJ, Le Roux I, Taljaard JJ. Combined use of pleural adenosine deaminase with lymphocyte/ neutrophil ratio. Increased specificity for the diagnosis of tuberculous pleuritis. Chest 1996;109:414-9.

7. Riantawan $\mathrm{P}$, Chaowalit $\mathrm{P}$, Wongsangiem $\mathrm{M}$, Rojanaraweewong P. Diagnostic value of pleural fluid adenosine deaminase in tuberculous pleuritis with reference to HIV coinfection and a Bayesian analysis. Chest 1999;116:97-103.

8. Reechaipichitkul W, Kawamatawong T, Teerajetgul Y, Patjanasoontorn B. Diagnostic role of pleural fluid adenosine deaminase in tuberculous pleural effusion. Southeast Asian J Trop Med Public Health 2001;32:383-9.

9. Lee YC, Rogers JT, Rodriguez RM, Miller KD, Light RW. Adenosine deaminase levels in nontuberculous lymphocytic pleural effusions. Chest 2001;120:356-61.

10. Jiménez Castro D, Díaz Nuevo G, Pérez-Rodríguez E, Light RW. Diagnostic value of adenosine deaminase in nontuberculous lymphocytic pleural effusions. Eur Respir J 2003;21:220-4.

11. Chen ML, Yu WC, Lam CW, Au KM, Kong FY, Chan AY. Diagnostic value of pleural fluid adenosine deaminase activity in tuberculous pleurisy. Clin Chim Acta 2004;341:101-7.

12. Baba K, Hoosen AA, Langeland N, Dyrhol-Riise AM. Adenosine deaminase activity is a sensitive marker for the diagnosis of tuberculous pleuritis in patients with very low CD4 counts. PLoS ONE 2008;3:e2788.

13. Garcia-Zamalloa A, Taboada-Gomez J. Diagnostic accuracy of adenosine deaminase and lymphocyte proportion in pleural fluid for tuberculous pleurisy in different prevalence scenarios. PLoS ONE 2012;7:e38729.

14. Küpeli E, Karnak D, Elgün S, Argüder E, Kayacan O. Concurrent measurement of adenosine deaminase and dipeptidyl peptidase IV activity in the diagnosis of tuberculous pleural effusion. Diagn Microbiol Infect Dis 2009;65:365-71.

15. Wu YB, Ye ZJ, Qin SM, Wu C, Chen YQ, Shi HZ. Combined detections of interleukin 27, interferon- $\gamma$, and adenosine deaminase in pleural effusion for diagnosis of tuberculous pleurisy. Chin Med J (Engl) 2013;126:3215-21.

16. Keng LT, Shu CC, Chen JY, et al. Evaluating pleural ADA, ADA2, IFN- $\gamma$ and IGRA for diagnosing tuberculous pleurisy. J Infect 2013;67:294-302.

17. Li M, Wang H, Wang X, Huang J, Wang J, Xi X. Diagnostic accuracy of tumor necrosis factor-alpha, interferongamma, interleukin-10 and adenosine deaminase 2 in differential diagnosis between tuberculous pleural effusion and malignant pleural effusion. J Cardiothorac Surg 2014;9:118.

18. Sharma SK, Banga A. Pleural fluid interferon-gamma and adenosine deaminase levels in tuberculosis pleural effusion: a cost-effectiveness analysis. J Clin Lab Anal 2005;19:40-6.

19. Ungerer JP, Oosthuizen HM, Retief JH, Bissbort SH. Significance of adenosine deaminase activity and its isoenzymes in tuberculous effusions. Chest 1994;106:337.

20. Andreasyan NA, Hairapetian HL, Sargisova YG, Mardanyan SS, Badalyan LT, Khanoyan AS. Activity of adenosine deaminase and its isoforms in pleural fluid in tuberculous pleuritis. Med Sci Monit 2002;8:CR708-12.

21. Yildiz PB, Yazar EE, Gorgun D, Secik F, Cakir G. Predictive role of adenosine deaminase for differential diagnosis of tuberculosis and malignant pleural effusion in Turkey. Asian Pac J Cancer Prev 2011;12:419-23.

22. Kawamatawong T, Panompong K, Kiatboonsri S, Khupulsup K. The appropriate cutoff level of pleural fluid adenosine deaminase activity by diazyme commercial kit for diagnosis pleural tuberculosis in Ramathibodi hospital. Chest 2008;134(Suppl 2):p55001.

23. Valdés L, San José E, Alvarez D, Valle JM. Adenosine deaminase (ADA) isoenzyme analysis in pleural effusions: diagnostic role, and relevance to the origin of increased ADA in tuberculous pleurisy. Eur Respir J 1996;9:747-51.

24. Strankinga WF, Nauta JJ, Straub JP, Stam J. Adenosine deaminase activity in tuberculous pleural effusions: a diagnostic test. Tubercle 1987;68:137-40.

25. Porcel JM, Esquerda A, Bielsa S. Diagnostic performance of adenosine deaminase activity in pleural fluid: a singlecenter experience with over 2100 consecutive patients. Eur J Intern Med 2010;21:419-23.

26. Ogata Y, Aoe K, Hiraki A, et al. Is adenosine deaminase in pleural fluid a useful marker for differentiating tuberculosis from lung cancer or mesothelioma in Japan, a country with intermediate incidence of tuberculosis? Acta Med Okayama 2011;65:259-63.

27. Light RW, Macgregor MI, Luchsinger PC, Ball WC Jr. Pleural effusions: the diagnostic separation of transudates and exudates. Ann Intern Med 1972;77:507-13.

28. Light RW. Parapneumonic effusions and empyema. Proc Am Thorac Soc 2006;3:75-80.

29. Davies HE, Davies RJ, Davies CW, BTS Pleural Disease Guideline Group. Management of pleural infection in adults: British Thoracic Society Pleural Disease Guideline 2010. Thorax 2010;65 Suppl 2:ii41-53.

30. Antunes G, Neville E, Duffy J, Ali N, Pleural Diseases Group, Standards of Care Committee, British Thoracic Society. BTS guidelines for the management of malignant pleural effusions. Thorax 2003;58 Suppl 2:ii29-38.

31. Feres MC, Martino MC, Maldijian S, Batista F, Gabriel Júnior A, Tufik S. Laboratorial validation of an automated assay for the determination of adenosine deaminase activity in pleural fluid and cerebrospinal fluid [in English, Portuguese]. J Bras Pneumol 2008;34:1033-9.

32. Wu YH, Zhao GW, Wang XF, Wang MS. Pleural effusion adenosine deaminase is not accurate in diagnosis of pediatric tuberculous pleural effusion: a retrospective study. Eur Rev Med Pharmacol Sci 2015;19:1706-10.

33. Neves DD, Dias RM, Cunha AJ. Predictive model for the diagnosis of tuberculous pleural effusion. Braz J Infect Dis 2007;11:83-8.

34. Chang KC, Leung CC, Yew WW, Tam CM. Standard anti-tuberculosis treatment and hepatotoxicity: do dosing schedules matter? Eur Respir J 2007;29:347-51.

35. Chang KC, Leung CC, Yew WW, Lau TY, Tam CM. Hepatotoxicity of pyrazinamide: cohort and case-control analyses. Am J Respir Crit Care Med 2008;177:1391-6. 